\title{
国国pubvet
}

https://doi.org/10.31533/pubvet.v15n01a721.1-5

\section{Uso de imunonutriente para tratamento de episódio diarreico em cão}

\author{
Katherinne Barth Wanis Figueiredo ${ }^{19}$, Juliana de Abreu Pereira ${ }^{2 *} \mathbb{9}$, Natascha Jenifer \\ Morante $^{19}$, Thaís Karoline Pereira ${ }^{1}$, Adriana Bertelli Melzer ${ }^{1}$, Darlene Luciana Guse Kaiser ${ }^{1}$ \\ ${ }^{I}$ Discente do curso de Medicina Veterinária da Universidade Sociedade Educacional de Santa Catarina (UNISOCIESC), campus Joinville - SC, Brasil \\ ${ }^{2}$ Docente do curso de Medicina Veterinária da UNISOCIESC, campus Joinville. Joinville - SC, Brasil. \\ *Autor para correspondência, E-mail: juliana.abreu@unisociesc.com.br
}

\begin{abstract}
Resumo. A diarreia é considerada um sinal bastante comum na clínica de animais de companhia, sendo que o uso de nutrientes imunomoduladores no tratamento deste quadro é considerado uma nova modalidade terapêutica cada vez mais utilizada por médicos veterinários. A suplementação dietética de probióticos, prebióticos e L-glutamina têm mostrado efeitos benéficos no controle e tratamento da diarreia em humanos. Pelas poucas descrições de utilização de imunonutrientes na literatura médico veterinária, este trabalho teve como objetivo relatar o caso de um cão da raça Poodle, fêmea, idade de 13 anos, com quadro de diarreia, submetido ao tratamento com glutamina por via oral durante 5 dias, o que resultou em remissão completa do episódio diarreico.
\end{abstract}

Palavras-chave: glutamina, trato gastrointestinal

\section{Use of immunonutriente to treat a diarrheic episode in a dog}

\begin{abstract}
Diarrhea is considered a very common sign in the pet clinic, and the use of immunomodulatory nutrients in the treatment of this condition is considered a new therapeutic modality increasingly used by veterinarians. Dietary supplementation of probiotics, prebiotics, and L-glutamine has shown beneficial effects in the control and treatment of diarrhea in humans. Due to the few descriptions of the use of immunonutrients in the veterinary literature, this study aimed to report the case of a female Poodle dog, aged 13 years, with diarrhea, submitted to oral glutamine treatment for 5 days, which resulted in the complete remission of the diarrheal episode.
\end{abstract}

Keywords: glutamine, gastrointestinal tract

\section{Uso de imunnonutrientes para el tratamiento de episodio diarreico en perro}

Resumen. La diarrea se considera un signo muy común em la clínica de mascotas, y el uso de nutrientes inmunomoduladores en el tratamiento de esta afección se considera una nueva modalidad terapéutica cada vez más utilizada por los médicos veterinarios. La suplementación dietética de probióticos, prebióticos y L-glutamina han mostrado efectos beneficiosos em el control y tratamiento de la diarrea en humanos. Debido a las pocas descripciones del uso de inmunonutrientes em la literatura veterinaria, este estudio tuvo como objetivo informar el caso de una perra, de 13 años, con diarrea, sometida a tratamiento oral de glutmaina durante 5 días, lo que resultó em la remisión completa del episodio diarreico.

Palabras clave: glutamina, tracto gastrointestinal 


\section{Introdução}

A diarreia é um sinal clínico frequente na clínica de animais de companhia, sendo caracterizada pela alteração na consistência das fezes, associada ou não ao aumento da frequência de evacuação (Brandt et al., 2015). Seja qual for o fator desencadeante da diarreia, a principal consequência é a redução das vilosidades intestinais, levando principalmente à má absorção de nutrientes, desidratação e perda de peso (Valente et al., 2011; Volkmann et al., 2017). Comumente para o tratamento são realizadas intervenções dietéticas e farmacológicas, além da manipulação terapêutica da microbiota intestinal através do uso de antibióticos, probióticos e prebióticos (Jergens \& Simpson, 2012).

A suplementação dietética de probióticos, prebióticos e L-glutamina têm mostrado efeitos benéficos no controle e tratamento da diarreia (Allen et al., 2010; Holecek, 2013; Pereira \& Ferraz, 2017), pois auxiliam na redução do tempo de recuperação, melhoram a resposta imune, contribuem para o equilíbrio da microbiota intestinal, alteram condições físico-químicas do cólon com diminuição do pH, estimulam o crescimento de bactérias benéficas do colón, diminuem a translocação bacteriana, ajudam na formação do bolo fecal e estimulam o crescimento das vilosidades intestinais, o que aumenta a capacidade absortiva (Delcenserie et al., 2008; Flesch et al., 2014; Xavier et al., 2009).

A glutamina, também conhecida como L-glutamina, é um aminoácido não essencial, sendo o mais abundantemente encontrado no organismo animal e em várias espécies de animais, sendo considerado um aminoácido de grande importância para a manutenção da mucosa e da barreira intestinal (Ferreira et al., 2017). Este imunonutriente é amplamente utilizado para o tratamento de pacientes na medicina humana e seu uso é muito comum em pacientes críticos, tratamento de distúrbios de TGI, pacientes oncológicos, recuperação e ganho de massa muscular.

Estudos têm mostrado o efeito benéfico da glutamina sob o TGI, demonstrando o efeito do uso deste aminoácido no tratamento de diarreias e enfermidades intestinais (Kucuktulu et al., 2013). Diante do efeito já comprovado em literatura, este trabalho teve por objetivo descrever o caso de um cão com diarreia tratado com glutamina por via oral.

\section{Relato de caso}

Um cão da raça Poodle, com 13 anos de idade, fêmea, branca, castrada, com peso corporal de 6,8 kg; foi levado para atendimento em consultório veterinário. Conforme relato da proprietária, o animal apresentava anorexia e quadro de diarreia com quatro dias de evolução. A paciente apresentava diagnóstico prévio de dermatite atópica canina (DAC) desde 2012 e pancreatite crônica (PC) desde 2015. Para controle da DAC, o animal fazia uso continuado de deflazacort manipulado na dose de 0,22 $\mathrm{mg} / \mathrm{kg}$; uma cápsula a cada 5 dias e ração com baixo teor de gordura (Vet Life Obesity \& Diabetic Canine - Farmina Pet Foods - Bragança Paulista/ São Paulo - 160 g ao dia, fracionados em duas refeições de $80 \mathrm{~g}$, administradas a cada 12 horas).

Além disso, os exames de imagem revelaram pâncreas com dimensões aumentadas, medindo cerca $1,91 \mathrm{~cm}$ de largura; hipoecóico e ecotextura grosseira, sendo as imagens sugestivas de pancreatopatia crônica. Além dessas alterações pancreáticas, foram presentes alterações hepáticas (hepatomegalia, contorno preservado, textura homogênea e ecogenicidade difusamente reduzida; sendo essas imagens sugestivas de hepatopatia aguda), de vesícula biliar (repleção moderada, conteúdo anecóico, com presença de lama biliar ecoicas em quantidade moderada e paredes normoespessas; imagens sugestivas de colestase) e estômago (estratificação parietal evidenciando a camada submucosa e espessura da parede aumentada, medindo cerca de $0,56 \mathrm{~cm}$; sendo a imagem sugestiva de gastropatia). Apesar de todas as alterações de imagem presentes no trato gastrointestinal, a paciente permaneceu clinicamente estável do quadro de pancreatite, não sendo correlacionado ao episódio diarreico.

Ao exame clínico, o animal apresentava estado geral bom, desidratação estimada em 4\%, temperatura normal, mucosas normocoradas, frequências cardíaca e respiratória normais, ruídos gastrointestinais normais, tempo de preenchimento capilar normal; leve distensão e dor abdominal a palpação.

Conforme pode ser observado na Tabela 1, a paciente possuía exames recentes, os quais não possuíam alterações e não justificavam o quadro clínico. 
Tabela 1. Resultados de exames laboratoriais apresentados durante a consulta.

\begin{tabular}{lcc}
\hline Parâmetro & Resultado & Referência $(13,14)$ \\
\hline Hematócrito $(\mathrm{Ht})$ & $33 \%$ & $37-55 \%$ \\
Hemoglobina $(\mathrm{Hb})$ & $11,2 \mathrm{~g} / \mathrm{dL}$ & $12-18 \mathrm{~g} / \mathrm{dL}$ \\
Volume corpuscular médio (VCM) & $73,99 \mathrm{fl}$ & $60-77 \mathrm{fl}$ \\
Concentração de hemoglobina corpuscular média (CHCM) & $33,9 \%$ & $32-36 \%$ \\
Proteína plasmática total (PPT) & $7,6 \mathrm{~g} / \mathrm{dL}$ & $5,5-8,0 \mathrm{~g} / \mathrm{dL}$ \\
Leucócitos totais & $6.800 \mathrm{~mm}^{3}$ & $6.000-17.000 \mathrm{~mm}^{3}$ \\
Metamielócitos & $0 / \mathrm{mm}^{3}$ & $0 / \mathrm{mm}^{3}$ \\
Bastões & $68 / \mathrm{mm}^{3}$ & $0-510 / \mathrm{mm}^{3}$ \\
Neutrófilos segmentados & $5.780 / \mathrm{mm}^{3}$ & $3.000-11.500 / \mathrm{mm}^{3}$ \\
Linfócitos & $680 / \mathrm{mm}^{3}$ & $1.000-4.800 / \mathrm{mm}^{3}$ \\
Monócitos & $204 / \mathrm{mm}^{3}$ & $150-1.350 / \mathrm{mm}^{3}$ \\
Eosinófilos & $68 / \mathrm{mm}$ & $100-1.250 / \mathrm{mm}^{3}$ \\
Basófilos & $0 / \mathrm{mm}^{3}$ & $0-170 / \mathrm{mm}^{3}$ \\
Plaquetas & $642.000 / \mu 1$ & $144.000-510.000 / \mu 1$ \\
Albumina & $2,51 \mathrm{~g} / \mathrm{dL}$ & $2,3-3,1 \mathrm{~g} / \mathrm{dL}$ \\
Alanina aminotransferase (ALT) & $81,5 \mathrm{U} / \mathrm{L}$ & $0-102 \mathrm{U} / \mathrm{L}$ \\
Aspartatoaminotransferase (AST) & $26,6 \mathrm{U} / \mathrm{L}$ & $23-66 \mathrm{U} / \mathrm{L}$ \\
Cálcio iônico & $3,49 \mathrm{mg} / \mathrm{dL}$ & $4,5-5,75 \mathrm{mg} / \mathrm{dL}$ \\
Creatinina & $0,51 \mathrm{mg} / \mathrm{dL}$ & $0,5-1,5 \mathrm{mg} / \mathrm{dL}$ \\
Uréia & $43,0 \mathrm{mg} / \mathrm{dL}$ & $10-40 \mathrm{mg} / \mathrm{dL}$ \\
Fosfatase Alcalina (FA) & $615,1 \mathrm{U} / \mathrm{L}$ & $20-150 \mathrm{U} / \mathrm{L}$ \\
Fósforo & $2,65 \mathrm{mg} / \mathrm{dL}$ & $2,9-5,3 \mathrm{mg} / \mathrm{dL}$ \\
\hline Valores erefer &
\end{tabular}

Valores de referência: Kaneko et al. (2008); Jain (1993).

Devido ao histórico clínico do animal, optou-se por iniciar suplementação com L-glutamina por via oral na dose de $0,25 \mathrm{~g} / \mathrm{kg}$ (Viana, 2014), a cada doze horas (totalizando $3 \mathrm{~g}$ ao dia) durante 5 dias. Foi realizada a diluição de $1,4 \mathrm{~g}$ de glutamina em $5 \mathrm{~mL}$ de água para facilitar a administração. Após o término do tratamento, o animal retornou para avaliação com total remissão do quadro clínico, conforme pode ser observado no aspecto das fezes da paciente (Figura 1).
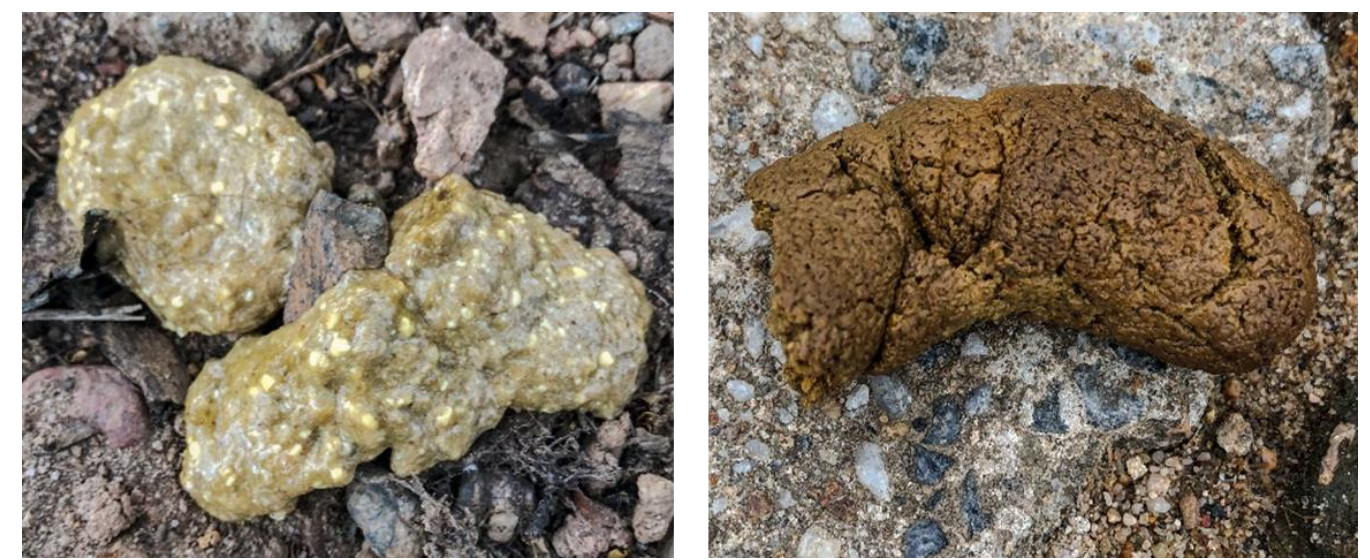

Figura 1. Aspecto das fezes do animal antes e após o tratamento. À esquerda podem ser observadas fezes acólicas, com aspecto pastoso e granulações. À direita, observam-se fezes de coloração amarronzada, aspecto modelado e sem granulações presentes.

\section{Discussão}

Em animais de companhia, a diarreia é um sinal clínico normalmente associado à presença de vírus, parasitos, bactérias, intoxicações por agentes tóxicos ou alimentares. Neste caso, o animal em questão realizava controle periódico de parasitos, o que removeu a suspeita de doença de caráter parasitário. Além disso, alterações nos exames laboratoriais não foram correlacionadas a enfermidades infecciosas, 
uma vez que pacientes tratados por períodos prolongados com corticóides podem apresentar características de imunossupressão, representadas no hemograma da paciente pela linfopenia e eosinopenia. Anemia discreta, distúrbios discretos do metabolismo mineral (hipocalcemia e hipofosfatemia discretas) e os elevados teores da enzima fosfatase alcalina podem ser correlacionados à outras enfermidades a esclarecer, sendo diagnósticos diferenciais o hiperparatireoidismo secundário renal e hiperadrenocorticismo canino.

A diarreia é uma complicação comum em pacientes com pancreatite aguda ou crônica; sendo que, na maioria dos casos, este sinal clínico ocorre devido à ingestão de uma dieta rica em lipídios ou pela associação de um quadro infeccioso e/ou inflamatório. Neste caso, o animal apresentava diagnóstico de PC há quatro anos e realizava manejo do quadro por meio do controle dietético com ração hipolipídica (Watson et al., 2013). Devido à lesão do órgão pela PC, sua capacidade de digestão de lipídios é reduzida, podendo causar dor, distensão abdominal e diarreia (Harris et al., 2017; Xenoulis, 2015); sinais esses que vem de encontro aos apresentados pelo animal em questão no momento da primeira avaliação clínica.

O uso da glutamina é seguro em animais conforme já descrito por outros estudos (Costa et al., 2009). A glutamina é utilizada pelos enterócitos como umas das principais fontes de energia, sendo que a depleção deste nutriente pode levar à alteração da barreira do epitélio digestivo, o que aumenta o risco de translocação bacteriana e sepse (Pacífico et al., 2005). O uso da glutamina em pacientes com diarreia tem como principal objetivo o aumento da espessura da mucosa intestinal, visto que este já está lesionado pelo quadro; e evitar a translocação bacteriana, o que na maioria das vezes contribui para a melhora do quadro diarreico.

\section{Conclusão}

Apesar da pouca utilização da glutamina na medicina veterinária, pode-se observar rápida melhora clínica da paciente descrita neste caso. Os resultados promissores obtidos reiteram os benefícios da incorporação deste imunoestimulante no tratamento de distúrbios gastrointestinais em animais.

\section{Referências}

Allen, S. J., Martinez, E. G., Gregorio, G. V, \& Dans, L. F. (2010). Probiotics for treating acute infectious diarrhoea. Cochrane Database of Systematic Reviews, 11. https://doi.org/10.1002/14651858.CD003048.pub3

Brandt, K. G., Antunes, M. M. C., \& Silva, G. A. P. (2015). Acute diarrhea: evidence-based management. Jornal de Pediatria (Versão Em Português), 91(6, Supplement 1), S36-S43. https://doi.org/10.1016/j.jpedp.2015.09.006

Costa, P. R. S., Conceição, L. G., \& Lopes, M. A. F. (2009). Nutrição enteral precoce com glutamina em cães com gastrenterite hemorrágica pelo parvovirus canino. Arquivo Brasileiro de Medicina Veterinária e Zootecnia, 61(5), 1251-1253. https://doi.org/10.1590/S0102-09352009000500035

Delcenserie, V., Martel, D., Lamoureux, M., Amiot, J., Boutin, Y., \& Roy, D. (2008). Immunomodulatory effects of probiotics in the intestinal tract. Current Issues in Molecular Biology, 10(1/2), 37 .

Ferreira, V. F., Silva, V. L. D., Ferraz, H. T., Bueno, P. C., \& Viu, M. A. O. (2017). Nutrição clínica de cães hospitalizados: Revisão. Pubvet, 11(9), 901-912. https://doi.org/10.22256/pubvet.v11n9.901912

Flesch, A. G. T., Poziomyck, A. K., \& Damin, D. C. (2014). O uso terapêutico dos simbióticos. ABCD. Arquivos Brasileiros de Cirurgia Digestiva (São Paulo), 27(3), 206-209. https://doi.org/10.1590/S010267202014000300012

Harris, J. P., Parnell, N. K., Griffith, E. H., \& Saker, K. E. (2017). Retrospective evaluation of the impact of early enteral nutrition on clinical outcomes in dogs with pancreatitis: 34 cases (2010-2013). Journal of Veterinary Emergency and Critical Care, 27(4), 425-433. https://doi.org/10.1111/vec.12612

Holecek, M. (2013). Side Effects of Long-Term Glutamine Supplementation. Journal of Parenteral and Enteral Nutrition, 37(5), 607-616. https://doi.org/10.1177/0148607112460682

Jain, N. C. (1993). Essentials of Veterinary Hematology (1st ed.). Wiley-Blackwell. 
Jergens, A. E., \& Simpson, K. W. (2012). Inflammatory bowel disease in veterinary medicine. Front Biosci (Elite Ed), 4(4), 1404-1419.

Kaneko, J. J., Harvey, J. W., \& Bruss, M. L. (2008). Clinical biochemistry of domestic animals (6th Ed. (ed.); Vol. 1). Academic press.

Kucuktulu, E., Guner, A., Kahraman, I., Topbas, M., \& Kucuktulu, U. (2013). The protective effects of glutamine on radiation-induced diarrhea. Supportive Care in Cancer, 21(4), 1071-1075. https://doi.org/10.1007/s00520-012-1627-0

Pacífico, S. L., Leite, H. P., \& Carvalho, W. B. (2005). A suplementação de glutamina é benéfica em crianças com doenças graves? Revista de Nutrição, 18(1), 95-104. https://doi.org/10.1590/S141552732005000100009

Pereira, I. G., \& Ferraz, I. A. R. (2017). Suplementação de glutamina no tratamento de doenças associadas à disbiose intestinal. Revista Brasileira de Saúde Funcional, 1(1), 46.

Valente, I., Lima, R., Carvalho, C., Ferreira, H., Marcelino, F., Mota, C., Ramos, A., Duarte, C., Carneiro, F., \& Pereira, F. (2011). Diarreia Intratável: Doença de Inclusão das Microvilosidades num Recém-Nascido. Jornal Português de Gastrenterologia, 18(6), 298-302.

Viana, F. A. B. (2014). Guia terapêutico veterinário. In Lagoa Santa.

Volkmann, M., Steiner, J. M., Fosgate, G. T., Zentek, J., Hartmann, S., \& Kohn, B. (2017). Chronic Diarrhea in Dogs - Retrospective Study in 136 Cases. Journal of Veterinary Internal Medicine, 31(4), 1043-1055. https://doi.org/10.1111/jvim.14739

Watson, P. J., Archer, J., Roulois, A. J., Scase, T. J., \& Herrtage, M. E. (2013). Observational study of 14 cases of chronic pancreatitis in dogs. Veterinary Record Case Reports, 1(1), ec4912. https://doi.org/10.1136/vetreccr.c4912rep

Xavier, H., Souza, M. R., Liberali, R., \& Coutinho, V. F. (2009). Relação do consumo de glutamina na melhora do trato gastrointestinal-revisão sistemática. O papel da glutamina no trato gastrointestinal. RBONE-Revista Brasileira de Obesidade, Nutrição e Emagrecimento, 3(18).

Xenoulis, P. G. (2015). Diagnosis of pancreatitis in dogs and cats. Journal of Small Animal Practice, 56(1), 13-26. https://doi.org/10.1111/jsap.12274

Histórico do artigo:

Recebido: 8 de maio, 2020

Aprovado: 15 de junho, 2020.

Disponível online: 18 de novembro, 2020
Licenciamento: Este artigo é publicado na modalidade Acesso Aberto sob a licença Creative Commons Atribuição 4.0 (CC-BY 4.0), a qual permite uso irrestrito, distribuição, reprodução em qualquer meio, desde que o autor e a fonte sejam devidamente creditados. 